\title{
PIV investigation of cavitating flows around circular cylinders with hydrophobic coatings
}

\author{
K. Dobroselsky, A. Lebedev, A. Safonov, S. Starinskiy, V. Dulin* \\ ${ }^{1}$ Kutateladze Institute of Thermophysics, Novosibirsk, Russia \\ *corresponding author: vmd@itp.nsc.ru
}

The treatment of the hydrophobic properties of solid surfaces is considered as a passive method to reduce the drag in water flows (Rothstein, 2010) and to potentially affect the flow separation and vortex shedding (Sooraj et al., 2020). The manufacturing of surfaces with micro- and nano-scale roughness allows to extend the hydrophobicity towards superhydrophobicity with the contact angle close to $180^{\circ}$. In such conditions the solid surface is not wetted completely and the air-water interphase partially remains on the surface texture. This results in so-called flow slip effect. Therefore, a local phase transition during the flow cavitation or gas effervescence in near-wall low-pressure regions may additionally affect the slip effect for hydrophobic surfaces. The present work is focused on the comparison between cavitating and noncavitating flows around circular cylinders with lateral sectors with hydrophobic and non-hydrophobic coatings. The experiments are performed in a water tunnel, which consists of a water outgassing and cooling/heating section, honeycomb, contraction section, test section and diffuser. The water flow is driven by an electric pump, providing a bulk velocity up to $10 \mathrm{~m} / \mathrm{s}$ in the transparent test section with $1 \mathrm{~m}$ length and $80 \times 150 \mathrm{~mm}^{2}$ rectangular cross-section. The facility is equipped with an ultrasonic flowmeter, temperature and pressure sensors. Besides, the static pressure inside the water tunnel can be varied by using a special shaft section. The measurements are performed by using high-repetition and low-repetition PIV systems. The former is used for the analysis of large-scale flow dynamics in the wake region, whereas the latter one is used for high-resolution measurements in near-wall regions by using a long-distance microscope. The Reynolds number based on the bulk velocity of the flow, diameter of the cylinders $(D=26 \mathrm{~mm})$ and kinematic viscosity of the water is varied up to $2 \times 10^{5}$.

The used cylinders were made of stainless steel and covered by fluoropolymer to alter hydrophobic properties by using a hot wire chemical vapor deposition method (see Safonov et al., 2018). To cover the cylinders with an uniform coating layer with the thickness of approximately $500 \mathrm{~nm}$, they were mounted on a rotating holder inside a vacuum deposition chamber with the pressure of 0.5 torr. The hexafluoropropylene oxide $\mathrm{C}_{3} \mathrm{~F}_{6} \mathrm{O}$ served as a precursor gas for the fluoropolymer film. The precursor was activated by nichrome wires at temperature of $640{ }^{\circ} \mathrm{C}$. During the deposition, the substrate temperature was about $30^{\circ} \mathrm{C}$. The contact angle for the water droplets on the coated surface was $124^{\circ}$ as measured by a DSA-100 KRUSS device (see Figure 1). Afterwards the fluoropolymer coating on the cylinders was locally removed to obtain lateral sectors with non-hydrophobic properties (with the contact angle of $88^{\circ}$ ). It was done by ablating the fluoropolymer coating by using the second harmonic $(532 \mathrm{~nm})$ of a pulsed Nd:YAG laser. The laser radiation was focused on the cylinder surface with the effective spot of $0.9 \mathrm{~mm}^{2}\left(\mathrm{e}^{2}\right.$ criterion). The laser fluence of the translated spot was $0.3 \mathrm{~J} / \mathrm{cm}^{2}$. The flow around four kinds of cylinders with the diameter of $26 \mathrm{~mm}$ were studied, viz., the stainless-steel cylinders, entirely coated by the fluoropolymer and without the coating. For the other two the fluoropolymer coating at the lateral surface of $180^{\circ}$ and $90^{\circ}$ sectors was removed (see Figure 1).

(a)

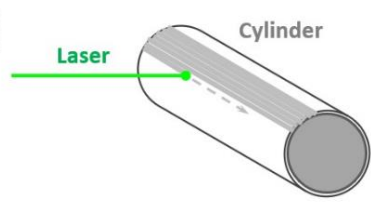

(c)

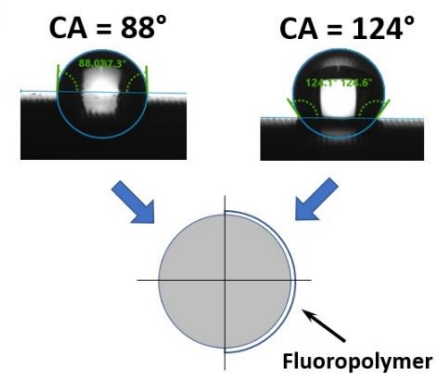

(b)

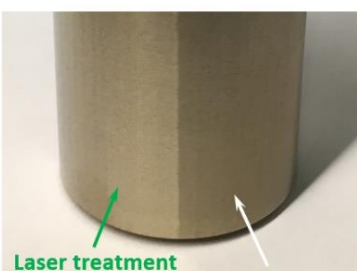

Laser treatment

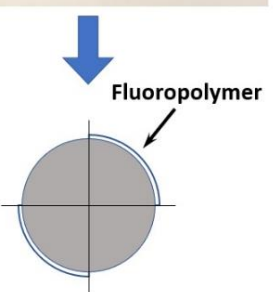

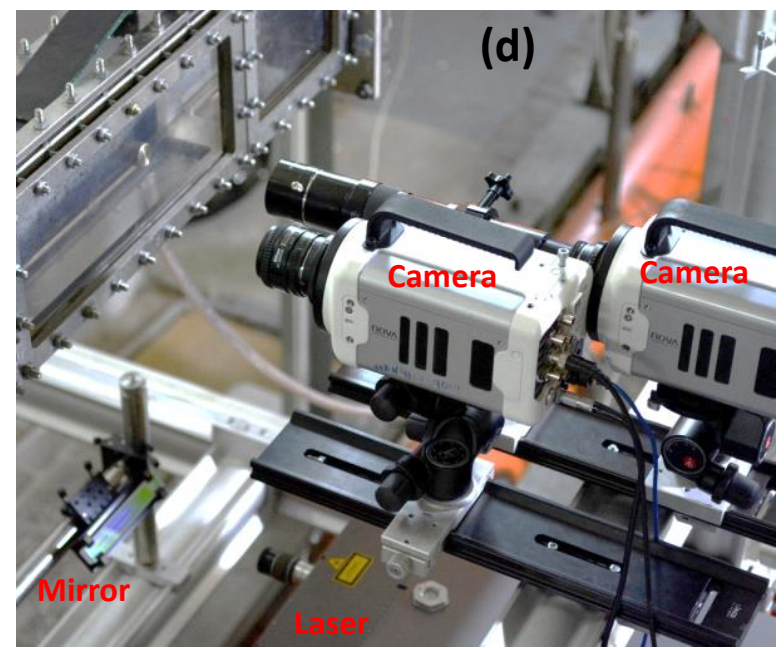

Figure 1 a) The scheme of nanosecond laser removal of fluoropolymer coating from stainless steel cylinder surface. b) The image of treated cylinder. c) The measurements of contact angles. d) Photo of the experimental setup 
The velocity field measurements were taken by using two independent PIV systems. A low-speed PIV system consisted of a pulsed dual-head Nd:YAG laser Polis $(50 \mathrm{~mJ}$ pulses with the repetition rate of $7 \mathrm{~Hz}$ ) and a CCD camera (Bobcat ImperX with the images of $2048 \times 2048$ pixels). A high-repetition system consisted of a pulsed Nd:YAG laser Photonics DM and two high-speed cameras Photron Nova S12 with different field of views (Figure 2). The laser provided $8 \mathrm{~mJ}$ pulses with repetition rate of $10 \mathrm{kHz}$. At this acquisition rate the high-speed cameras provided $1024 \times 1024$ pixel images. These cameras were also used for the high-speed visualization of the cavitating flows. The laser beams were converted into laser sheets by using cylindrical and spherical lenses. The laser sheet thickness for the low-repetition PIV system was less than $0.8 \mathrm{~mm}$. The lasers and cameras were synchronized by TTL signals. The PIV images were processed by using an in-house software ActualFlow. For the low-speed PIV an adaptive cross-correlation algorithm was used with the final interrogation area size of $32 \times 32$ pixels and $50 \%$ spatial overlap rate. For the high-repetition PIV measurements a multi-frame adaptive PIV algorithm, similar to that by Sciacchitano et al. (2012), was used. Figure 3 shows the example of the time averaged velocity field and visualisation of the flow separation for the cylinder with different orientation of the $180^{\circ}$ sector coating. The coating delays the flow separation and affects the shape of the wake.
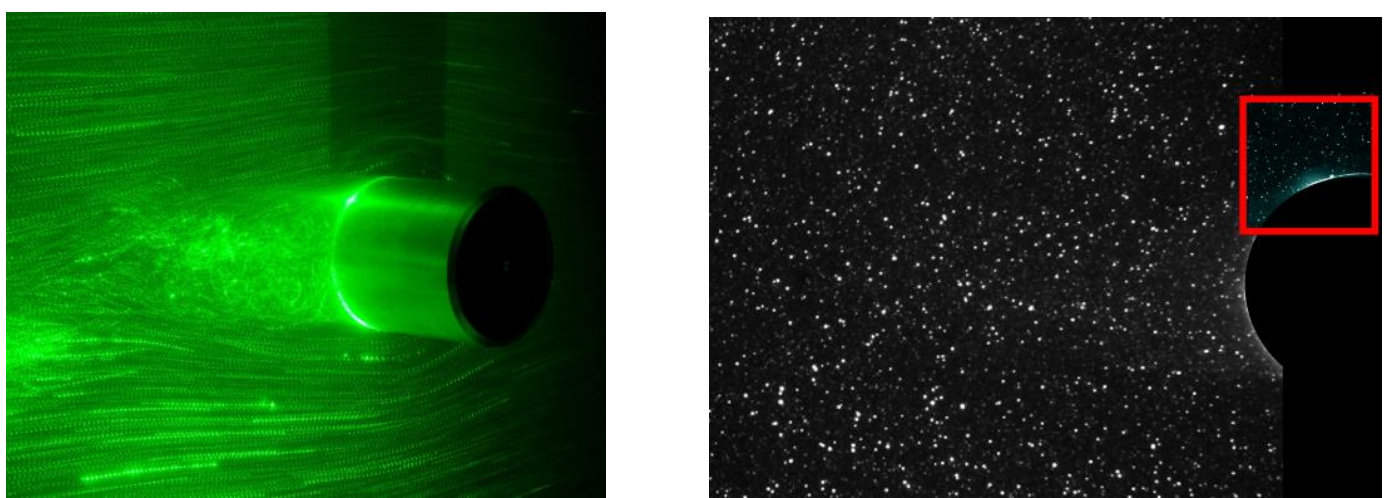

Figure 2 The photographs of the test section during the PIV measurements and the example of the PIV images by two cameras with different fields of view
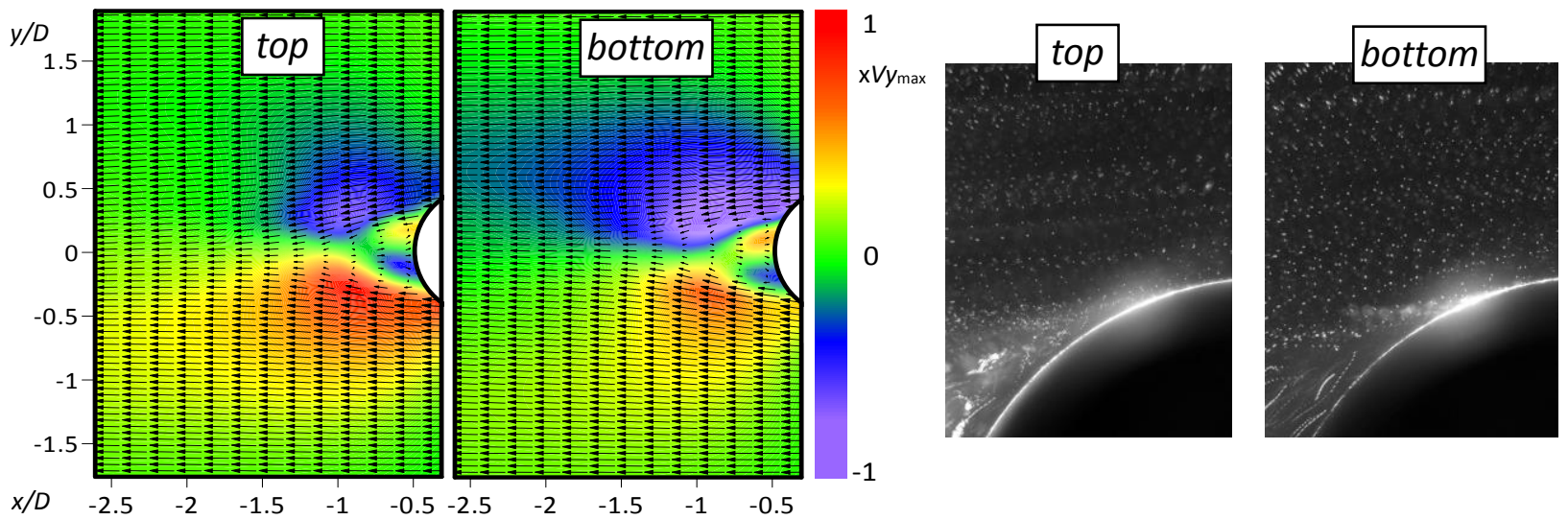

Figure 3 The examples of time-averaged velocity field and visualization of flow separation for a cylinder with superhydrophobic coating from the top and bottom for the Reynolds number of $2 \times 10^{5}$ (without cavitation)

The work was supported by Russian Government project 075-15-2019-1888 (supervised by Prof. C. Markides).

\section{References}

Rothstein JP (2010) Slip on superhydrophobic surfaces. Annual Review of Fluid Mechanics 42, 89-109

Sooraj P, Ramagya MS, Khan MH, Sharma A and Agrawal A (2020) Effect of superhydrophobicity on the flow past a circular cylinder in various flow regimes Journal of Fluid Mechanics 897, A21

Safonov AI, Sulyaeva VS, Gatapova EY, Starinskiy SV, Timoshenko NI and Kabov OA (2018) Deposition features and wettability behavior of fluoropolymer coatings from hexafluoropropylene oxide activated by NiCr wire Thin Solid Films 653, 165-172

Sciacchitano A, Scarano F, Wieneke B (2012) Multi-frame pyramid correlation for time-resolved PIV. Experiments in Fluids 53, 1087-1105 